\title{
Analysis on Key Points and Trend of 4G Mobile Communication Technology
}

\author{
Dong $\mathrm{Xu}^{1, \mathrm{a}}$ \\ ${ }^{1}$ Chongqing College of Electronic Engineering, Chongqing, China, 401331 \\ ${ }^{\mathrm{a} e m a i l,}$
}

Keywords: 4G Mobile Communication; Technical Points; Development Trend

\begin{abstract}
With the rapid development of information technology, communication technology control measures and operational mechanism has also been a leap-forward development, both from the communication technology model or the application of the scale of the industry have made significant progress. After the 21st century, the communication industry has developed the mobile communication technology. Compared with the 3G technology, the application of the 4G technology has certain advantages. It is the technical upgrading on the basis of the traditional communication technology. It is more convenient and quick to develop the communication industry in China. It has a driving force and significance, but also has important social value. This paper analyzes and discusses the key points and development trend of 4G mobile communication technology, and promotes the industry progress and technology upgrading.
\end{abstract}

\section{Introduction}

Under the information technology, the level of mobile communication technology has been improved obviously. The development of the communication industry is facing both new opportunities and certain challenges. With the development of $4 \mathrm{G}$ mobile communication technology, the communication business of China has been promoted. At the same time, mobile communication enterprises are faced with more intense market competition in the development process, so the communication industry needs to have some forward-looking awareness and crisis awareness, in order to promote Chinese mobile communication technology to get better development [1]. Therefore, the development of 4G mobile communication technology needs to coincide with the development of the times, and some advanced international advanced technology to master, in order to promote the development of $4 \mathrm{G}$ mobile communication technology, which is the new era of Chinese communications industry's new mission.

\section{Analysis of 4G Mobile Communication Technology}

The Meaning of 4G Technology. On the definition of 4G mobile communication technology the industry has not described it in detail, which mainly refers to the fourth generation of Chinese mobile communication technology, but for its lack of technical functions described and defined in detail. From the current development of $4 \mathrm{G}$ mobile communication technology in China, it is an inevitable trend in the development and social development of the communication industry. The meaning of 4G mobile communication technology is analyzed, and its function is first interpreted. First of all, 4G technology to break the time and space constraints, in the practical application process and supervision and management mechanism need to use network communications projects, the network system direct access and accessibility access, and 2G, 3G technology, 4G technology network has a fast, complete model and other advantages, while the overall mobile technology and business also has a variety of features [2]. Secondly, in the establishment and application of the technical system, the need for comprehensive management and centralized management of specific issues, 4G mobile communication technology on other applications and networks can produce adaptation for the communications industry to lay the foundation for business upgrades. At the same time, 4G mobile communication technology is based on 2G technology and 3G technology, in the operating mechanism has obvious differences, $4 \mathrm{G}$ operating system is the application of routing 
technology as the main constituent network architecture, through the establishment of the core network to ensure that the management model and mobile effects to upgrade. Compared with the unified network, 4G mobile communication technology has similarity, to ensure that the wireless connection, wired connection and mobile management and other functions of the optimization and upgrading [3]. In the establishment of application system will be divided into ATM and IP packet two modes, so as to promote the overall technical structure upgrade, wireless access point in the management structure of the application process, the wireless access point of the variability characteristics, the user can always intervene in the network and in the communication process to provide the effect of conversion. One of the most critical projects is the core network, so it is necessary to real-time management of the core network to ensure the stability of the location of the management model, and the identity of the user to identify.

The Features of 4G Mobile Communication Technology. 4G technology is not only efficient, the overall technical application model is more flexible, in the management structure and intelligent technology applications, in order to ensure the rational allocation of resources, should be in different channel conditions and the environment to apply intelligent signal processing to ensure that the signal conventional transceiver to ensure that the communication technology model to establish the system, and to ensure that the effect of treatment to ensure that the overall application system adaptability and intelligence [4]. 4G mobile communication technology in the establishment of the actual application mechanism to provide users with service models to ensure that different devices and users interdependent, so as to meet the actual needs of different users can be involved in real-time equipment, and to provide users with high-speed information service technology and communication, and has an autonomous network structure to meet the processing needs of different users, to achieve a dynamic management model has laid a solid foundation.

The Structure of 4G Technology Network. 4G technology network structure is more complex, which mainly contains four levels, namely, the physical layer, the middle layer, application layer and data transmission layer, in order to achieve better data transmission, improve data transmission quality of service, you need to apply Layer of the network to focus on control, in order to achieve technological optimization and upgrading [5].

\section{The Points of 4G Mobile Communication Technology}

The OFDM Technology. OFDM technology, also known as orthogonal frequency division multiplexing technology, its main function is to divide a channel into multiple orthogonal subchannels, and high-rate data signals into low-speed sub-data stream, in order to achieve the relevant data flow in the transmission and operation on each subchannel, and ensuring the overall information dissemination efficiency and stability. The spectrum is more efficient in the technology, compared with the traditional structure of the serial rate is twice or more than the serial rate [6]. In addition, in the operation of OFDM technology because of its own anti-drop ability, can use a number of subcarriers for data transmission, in order to achieve the overall transport efficiency and effectiveness of the impulse noise has a certain resistance, its adjustment mechanism and Transmission speed also has a certain practical value, so that the application of the modulation method to improve the effect, but also improve the channel processing and loading algorithm measurement effect, the information transmission rate has been significantly improved to ensure that the application mechanism optimization and upgrading.

SA Technology. In the 4G mobile communication technology to obtain a wide range of technical system is the SA technology model, also known as smart antenna technology, the technology can effectively inhibit the signal interference to ensure that the processing effect, but also to ensure the integrity of the application mechanism for automatic tracking technology Upgrade to provide new impetus to protect the digital wave adjustment effect consistent with the relevant standards, but also can be adjusted to the special function to ensure that the application of technology effects and processing effects [7].

SDR Technology. SDR technology is also known as software radio technology, the technology is a new technology model, but also micro-electronics technology, modern communication theory is 
an important basis for the development of SDR technology, mainly by means of microelectronics technology to deal with digital signals in $4 \mathrm{G}$ In the communication system, it is necessary to ensure that the mobile terminal equipment is suitable for various types of interfaces, and can be roamed seamlessly in different network environments, and can be carried out with different service types in order to realize the ideal state of access to the network in any form of anytime, Free conversion [8]. This also shows that the various systems in the $4 \mathrm{G}$ system will become more complex, and in the 4G mobile communication system to join the radio technology, can accelerate the optimization and upgrading of 4G communication technology, communication transmission more convenient and effective, in order to achieve the overall technical model to optimize and implement, to ensure that 4G mobile communication technology in the model building process to establish an open and standard hardware structure, allowing multi-operator intervention, which reflects the unique nature of SDR technology. SDR technology with advanced new technology as the core, has great potential for development, but also has a high market value, mainly emphasizes the indirect platform hardware indirect, the different application software to re-configure and upgrade, in order to achieve different functions design ideas.

Ipv6 Technology. IPv6 technology is essentially a network address space, mainly in the mobile communication process for the transmission of data to provide a network address, and provide the network address is unique, while IPv6 technology can also automatically configure the communication network, and Access to a unique router, which in $4 \mathrm{G}$ mobile communication technology reflects the importance of IPv6 technology. The development of IPv6 technology is based on the establishment of IPv4 technology, the original technology to upgrade online, is a service system, the quality of mobile communications to provide a guarantee of stability.

\section{The Development Trend of 4G Mobile Communication Technology}

Anti-Jamming Technology. In the rapid development of science and technology at the same time, 4G mobile communication technology development is bound to the direction of intelligent development, and the current technology is facing the biggest problem is the electromagnetic interference problem, the mobile communication effect has an important impact, so only the development of new Anti-jamming technology, in the communication transmission in order to eliminate the interference of electromagnetic waves, the advantages of $4 \mathrm{G}$ technology to play out. At present, the interference suppression technology has been widely used in communication business both at home and abroad, and achieved good results, and anti-jamming technology is the key to interactive interference suppression technology, to ensure that $4 \mathrm{G}$ mobile communication technology from electromagnetic interference. In the application of $4 \mathrm{G}$ mobile communication technology, the use of interactive interference suppression technology, its in-depth study to ensure that 4G mobile communication technology in the transmission process is not subject to electromagnetic interference, thereby improving communication quality.

Identification Technology. 4G mobile communication technology is currently a large number of users, and a wide range of distribution, according to the survey found that the number of $4 \mathrm{G}$ mobile communications users increased year by year, when more than 800 million people. In order to meet the communication needs of many users, and fully embodies the humanization and intelligence of communication technology, we must strengthen the R \& D identification technology, many users to identify one by one to meet the different needs of users, and this as a research focus, the establishment of 4G mobile communication base stations, enhance system capacity, the user quickly and accurately identified, so that the quality of communication services.

Receiving Technology. To further promote 4G technology, we must ensure that the technology of environmental protection and energy-saving performance, in order to promote 4G mobile communication technology has been further developed and improved, it is necessary to build on the basis of 3G mobile communication technology to develop signal reception technology, reflecting its energy efficiency, thus Improve the advantages and competitiveness of 4G mobile communication technology [9]. 4G technology is used in embedded radio receivers, low power consumption, compared with traditional technology, its power consumption accounted for only one percent, so 
that greatly reduced energy damage, but also play a significant role in environmental protection, while receiving technology not only has the characteristics of low energy consumption, improve the stability and reliability of signal acceptance, more communication users are more popular technology, 4G technology has broad prospects for development.

Self-Healing Network Technology. The intelligent technology has been applied in 4G mobile communication technology. Its intelligent processing can deal with the overloading of base stations and node faults automatically. The other parts use the Q \& A device to correct and deal with the existing problems, So as to automatically eliminate the network failure, improve the quality of communication services.

Wireless Access Network Technology. 4G mobile communication technology and 3G technology compared to the lower cost, greater capacity, faster transmission speed, to accept a wide range, so 4G mobile communication technology development prospects are more extensive, to further promote the development of the communications industry, it is necessary on this basis to strengthen the development of radio technology, research and development of switching circuit converters, equipment to the network diversity, network architecture can make 4G, 3G and WLAN, for the next generation of Internet and mobile communication technology to make greater contributions to promote 4G mobile The development of communication technology.

The Upgrading of Ipv6 Technology. In the process of 4G mobile communication model establishment, IPv6 technology has also been upgraded and improved. In the establishment of technology model, network address space is needed to ensure that network communication equipment only has the only geological establishment of its communication relationship, to ensure the effectiveness of application system and automatic configuration of structure validity. In the IPv6 technology applications can provide high-level system services, and to ensure the application of mobility advantages, through IPv6 technology to ensure the stability of location changes and to ensure that the overall quality of mobile communications will not be affected to ensure that mobile communications technology services quality.

\section{Conclusion}

In short, 4G mobile communication technology for the development of the communications industry provides a new development opportunities, but also to the communications industry is facing major challenges, 4G mobile communication technology in the management mechanism and operational structure are reflected in the diversity of its technical applications the model is more humane and intelligent features, 4G mobile communication service quality level has also been significantly improved, give full play to the advantages of $4 \mathrm{G}$ technology for the development of mobile communications industry laid the foundation to provide effective protection. In the traditional communication technology on the basis of technological upgrading, more convenient and fast, the development of Chinese telecommunications industry has a role in promoting and important, but also has important social value.

\section{References}

[1] Yan Hai Shan. 4G mobile communication technology key points and development trends [J]. China's new communication, 2016, 18 (22): 18.

[2] Yan Ningxiao. 4G mobile communication technology points and development trends [J]. China's new communication, 2017, 19 (02): 40.

[3] Wang Liebin. 4G mobile communication technology analysis and development trend analysis [J]. Information Communication, 2017, (06): 212-213.

[4] Wang Xiaoxia. On 4G mobile communication technology key points of analysis and development trends [J]. China Information Technology, 2017, (07): 48-49. 
[5] Shizhu, Lu Zhiqiang. New period 4G mobile communication technology points and development trends [J]. Science and Technology, 2014, (03): 228. 